\title{
An efficient MFS model for the analysis of sonic crystals including fluid-solid interaction
}

\author{
P. G. Santos ${ }^{1}$, J. Carbajo ${ }^{2}$, L. Godinho ${ }^{1,3}$ \& J. Ramis ${ }^{2}$ \\ ${ }^{1}$ Department of Civil Engineering, University of Coimbra, Portugal \\ ${ }^{2}$ DFISTS, University of Alicante, Spain \\ ${ }^{3}$ Centre for Research in Construction Science (CICC), Portugal
}

\begin{abstract}
Sonic crystals are a relatively recent concept that is being explored for different applications. Several types of models exist in literature for their analysis, although in many cases they consider their elements to be rigid. In this work, the authors propose a hybrid numerical-analytical model based on the Method of Fundamental Solutions (MFS) to address the problem of sound scattering by a sonic crystal composed of non-rigid scatterers. This model would account for the full interaction between the acoustic medium and an array of elastic shell structures, which are assumed to be circular and filled with fluid. For that purpose, each scatterer is accounted for by means of a closed form solution, and the full set of scatterers is then coupled by means of the MFS. This strategy allows a compact description of the propagation medium while being very accurate and efficient from the computational point of view. Application examples are presented considering structures embedded in different acoustic media (air or water), and using the sonic crystal either as a waveguide or as a sound barrier.
\end{abstract}

Keywords: MFS, sonic crystals, fluid-solid interaction.

\section{Introduction}

The study of periodic structures for sound attenuation, namely sonic crystals, has been a topic of increasing interest in recent years. Sonic crystals get their name by analogy with ordered structures of semiconductor materials such as silicon crystals, whose feature of allowing certain energy waves to pass through and block others is transposed, in sonic crystals, into the capacity to prevent or limit the propagation of certain sound frequencies. Historically, it is generally considered 
that the first evidence that it was possible to achieve some effect of acoustic obstruction using structures in periodic arrays was derived fortuitously from a sculptural element, in the gardens of the Fundación Juan March in Madrid (Martínez-Sala et al. [1]). Since then, different aspects of the behavior of sonic crystals have been studied, some of which were essentially theoretical, as the influence of point defects (Wu et al. [2]) or the existence of waveguides in which the sound propagates with low attenuation (Vasseur et al. [3]). Practical uses of sonic crystals have been analyzed, and perhaps the most promising one is their use for the selective attenuation of sound, for example as traffic noise barriers (Sánchez-Pérez et al. [4]), since they can be a competitive solution when compared with classic noise barriers (Castiñeira-Ibáñez et al. [5]). Although a significant number of works has been published, the subject of sonic crystals is still under development and there are several issues that need further study. Recently a 2D approach to deal with the acoustic behavior of sonic crystals based on the MFS that considers the scatterers as rigid elements was proposed by Martins et al. [6]. The comparison between results provided by the numerical model and the ones provided by the Boundary Element Method (BEM) has shown good accuracy of the model as well its advantages in terms of computation times. Also the comparison between numerical and experimental simulations for a laboratory scale model made of PVC revealed good agreement (Martins et al. [7]). In this paper, an improvement of this previous approach is presented to additionally account for the elasticity of shell scatterers. In this model, the problem is divided in a series of subregions, one of them being the outer region, and the remaining ones, regions defined around each scatterer structure. As the fundamental/analytical solutions are known for each of those subregions, it becomes possible to establish a coupled model based on MFS, which accounts for the full interaction between the involved fluids and the solids that compose the scatterer structures, by just establishing the continuity of pressures and displacements along the boundaries connecting the subregions.

In summary, the method will be presented and validated as follow: first the theoretical formulation will be presented; a set of results obtained by the proposed model will then be verified against a finite element model and against the previous model developed by (Martins et al. [6]). In this point, different combinations of materials and shell thickness will be tested for two types of fluid, air and water. The comparison between results from these two models will show the influence of the scatterer's stiffness as the fluid characteristics varies in its attenuation behavior.

\section{Mathematical formulation}

\subsection{Governing equations}

Within the scope of this work, the 2D scattering of waves by cylindrical shell structures embedded within a fluid medium is analyzed. Thus, the governing equations of the problem correspond to the vectorial and scalar wave equations, respectively for the solid and for the fluid regions of the analysis domain. 
Considering a homogeneous, linear isotropic elastic domain with mass density $\rho_{S}$, shear wave velocity $\beta_{S}$ and compressional wave velocity $\alpha_{S}$, the propagation of elastic waves can be described by vectorial wave equation:

$$
\alpha_{\mathrm{S}}^{2}(\nabla \nabla \cdot \underline{\mathrm{u}})-\beta_{\mathrm{S}}{ }^{2} \nabla \times \nabla \times \underline{\mathrm{u}}=-\omega^{2} \underline{\mathrm{u}}
$$

where the vector $\underline{u}$ represents the displacement, $\omega$ is the circular frequency and, for a two-dimensional problem, $\nabla=(\partial / \partial x) \hat{i}+(\partial / \partial y) \hat{j} ; \hat{i}$ and $\hat{j}$ are the unit vectors along the $x$ and $y$ directions.

If the propagation medium is a fluid, with mass density $\rho_{f}$, the propagation is governed by the Helmholtz equation, which can be written as:

$$
\nabla^{2} \mathrm{p}+\mathrm{k}_{\mathrm{f}}^{2} \mathrm{p}=0
$$

where $p$ is the pressure and $k_{f}=\omega / \alpha_{f}$ is the wave number, with $\alpha_{f}$ being the speed of sound in the fluid medium; for this scalar equation, $\nabla^{2}=\left(\partial^{2} / \partial x^{2}\right)+\left(\partial^{2} / \partial y^{2}\right)$. Within this fluid medium, the displacements can be defined as a function of the first spatial derivative of $p$, and are given by:

$$
u_{x}=-\frac{1}{\rho_{\mathrm{f}} \omega^{2}} \frac{\partial p}{\partial x}, u_{y}=-\frac{1}{\rho_{\mathrm{f}} \omega^{2}} \frac{\partial p}{\partial y}
$$

\subsection{MFS formulation}

Consider a fluid medium and the presence of an arbitrary number of circular shell structures made of elastic materials, and filled with a fluid material. This configuration is depicted in Figure 1.

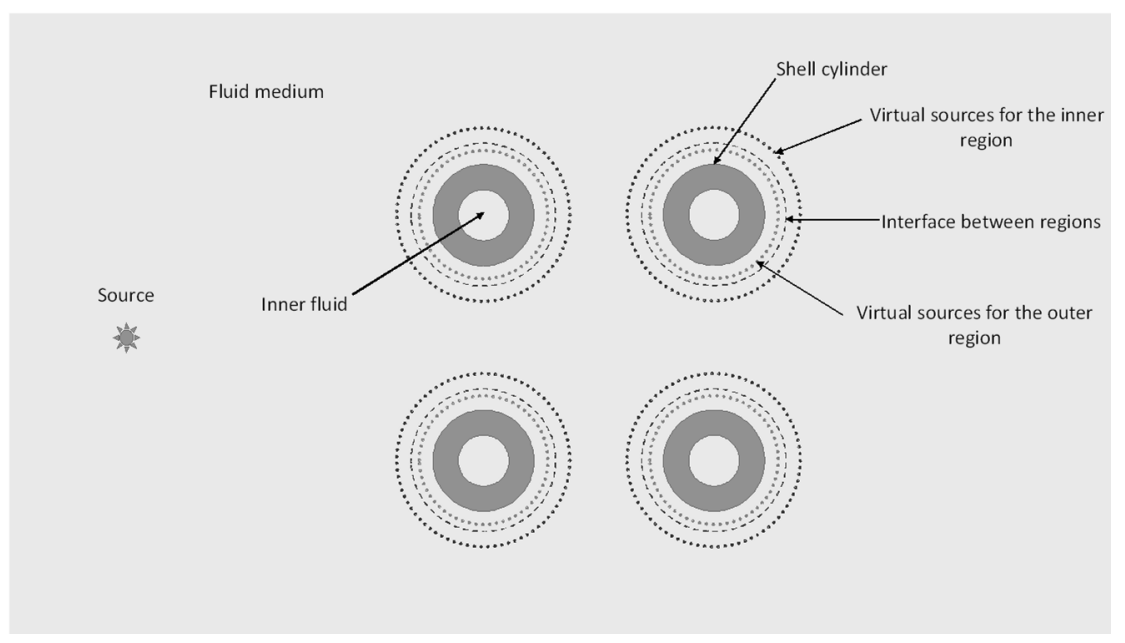

Figure 1: Schematic representation of the problem. 
Consider that, in the presence of $N R$ shell structures, the problem is divided in $N R+1$ subregions, one of them being the outer subregion (infinite fluid), and each of the $N R$ subregions is defined around the shell structure, as represented in Figure 1.

Knowing the fundamental solutions for each of the defined subregions, a coupled model can then be defined, accounting for the full interaction between the involved fluids and the solids that compose the shell structures. For that purpose, continuity of pressures and displacements along the boundaries connecting the regions (considering that each subregion of Figure 1 has its own inner and outer regions) must be enforced. Using the MFS, the acoustic field in the outer region can be defined by considering a number of virtual sources, $\sum_{\mathrm{j}=1}^{N R} N V S_{j}$ placed within all the subregions, and combining their effects in a linear manner as:

$$
p(\varkappa)=\sum_{j=1}^{N R} \sum_{l=1}^{N V S_{j}} a_{j, l} G\left(\varkappa, \varkappa_{j, l}^{\nu s}\right)+G\left(\varkappa, \varkappa_{0}\right)
$$

while for a point placed within the fluid of the $j$ th inner subregion, we have:

$$
p(\varkappa)=\sum_{l=1}^{N V S_{j}} b_{j, l} G^{\text {shell }}\left(\varkappa, \varkappa_{j, l}^{\nu s}\right)
$$

where $\mathcal{U}$ represents a point of coordinates $(x, y), \varkappa_{0}$ is the position of a real source illuminating the system, $\varkappa_{j, l}^{v s}$ is the position of each of the $N V S_{j}$ virtual sources placed within subregion $j, G\left(\varkappa, \varkappa_{0}\right)$ is the fundamental solution for the outer fluid at a point $\varkappa$ originated by a source positioned at $\varkappa_{0} ; G^{\text {shell }}\left(\varkappa, \varkappa_{0}\right)$ is the fundamental solution for each inner region, incorporating the full interaction between each shell structure and the outer and inner fluids; the coefficients $a_{j, l}$ and $b_{j, l}$ are unknowns that must be determined by conveniently establishing a system of equations, enforcing the continuity of pressures and displacements along each of the $N R$ boundaries separating the outer region from each inner region. If the boundary conditions are enforced at $N V S_{j}$ collocation points along the $k$ th boundary (as illustrated in Figure 1), the continuity equations along the $m$ th collocation point $\varkappa_{m}^{c, k}$ of that boundary can be written as:

$$
\begin{aligned}
& \sum_{j=1}^{N R} \sum_{l=1}^{N V S_{j}} a_{j, l} G\left(\varkappa_{m}^{c, k}, \varkappa_{j, l}^{v s}\right)+G\left(\varkappa_{m}^{c, k}, \varkappa_{0}\right)=\sum_{l=1}^{N V s_{k}} b_{k, l} G^{\text {shell }}\left(\varkappa_{m}^{c, k}, \varkappa_{k, l}^{v s_{\text {shell }}}\right) \\
& \sum_{j=1}^{N R} \sum_{l=1}^{N V S_{j}} a_{j, l} \frac{\partial}{\partial \vec{n}} G\left(\varkappa_{m}^{c, k}, \varkappa_{j, l}^{v s}\right)+\frac{\partial}{\partial \vec{n}} G\left(\varkappa_{m}^{c, k}, \varkappa_{0}\right)= \\
& \sum_{l=1}^{N V S_{k}} b_{k, l} \frac{\partial}{\partial \vec{n}} G^{\text {shell }}\left(\varkappa_{m}^{c, k}, \varkappa_{k, l}^{v s_{\text {shell }}}\right)
\end{aligned}
$$

A $N x N$ linear system of equations, with $=2 \times \sum_{j=1}^{N R} N V S_{j}$, is thus assembled. Once this system of equations is solved, one may obtain the pressure at any point outside the shell structures by applying equations (4) and (5). 
The fundamental solutions for both the external fluid and for the fluid-solidfluid system can be found in the literature, such as in Godinho et al. [8] and Godinho et al. [9].

It must be stressed that the coupling between subregions is enforced in fluid-fluid interfaces, at some distance from the interfaces with the solid media that constitutes the shell structures; using this strategy, the coupling can be performed in a region with smooth variations of the pressure, which greatly improves the performance of the MFS. Additionally, since the interface between subregions is virtual, it can assume a smooth shape, such as that of a circle, which has been demonstrated in previous works that leads to very accurate results (Godinho et al. [10]). Finally, if the fundamental solutions are computed analytically within each subregion, a further step can be given towards obtaining high accuracy.

\section{Verification of the model}

To verify the proposed coupled acoustic-elastodynamic MFS model, a comparison between the results computed with FEM (Finite Element Method) and MFS for a configuration including four scatterers embedded in water (with a density of $\rho_{f}=1000 \mathrm{~kg} / \mathrm{m}^{3}$ and allowing sound waves to propagate at $\alpha_{f}=1500 \mathrm{~m} / \mathrm{s}$ ) is considered. The four scatterers are assumed to be made of PolyVinyl Chloride (PVC), with a density of $\rho_{s}=1400 \mathrm{~kg} / \mathrm{m}^{3}$, a Young's modulus of $E=3.0146 \mathrm{GPa}$ and a Poisson's ration of $v=0.40622$, being $\beta_{s}=875 \mathrm{~m} / \mathrm{s}$ and $\alpha_{s}=2143 \mathrm{~m} / \mathrm{s}$. The scatterers have a fixed external radius of $0.1 \mathrm{~m}$, a thickness of $0.025 \mathrm{~m}$ and their centres are equally spaced $0.4 \mathrm{~m}$ between them. A point source is located $3 \mathrm{~m}$ in front of the scatterers and a square grid of receivers equally spaced $0.25 \mathrm{~m}$ is positioned $1 \mathrm{~m}$ behind them. Figure $2 \mathrm{a}$ exhibits the described configuration, and in Figure $2 b$ the FEM mesh around the scatterers is shown. A very refined mesh was used in this study in order to allow the analysis of higher frequencies (smaller wavelengths) of $5000 \mathrm{~Hz}$.
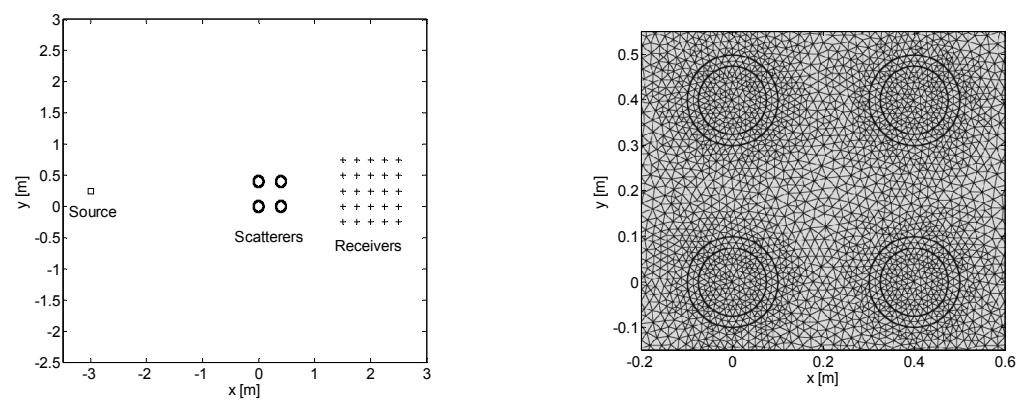

Figure 2: a) Configuration of the problem; b) detailed view of the FEM mesh. 
In Figure 3, the insertion loss calculated by the proposed MFS model and by the classic FEM approach are displayed, considering its average value over the grid of receivers.

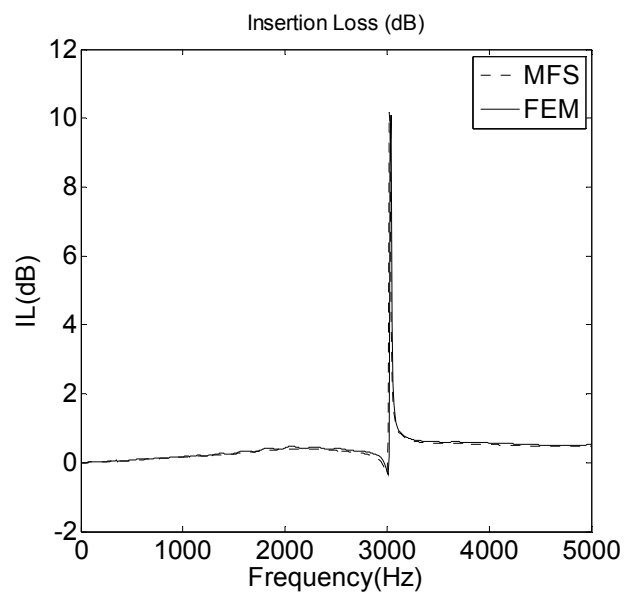

Figure 3: Comparison of the insertion loss provided by MFS and FEM models.

\section{Numerical simulations}

To better understand the effect of correctly modelling the elastic scatterers, results for insertion loss computed using the model proposed here were compared with those obtained using the methodology proposed by Martins et al. [6], in which just rigid cylinders are considered.

The basic layout analysed consisted on a system formed by an array of $10 \times 3$ shell cylinders arranged in a rectangular lattice configuration placed within a fluid medium and filled with the same fluid (Figure 4). The noise source was placed $3 \mathrm{~m}$ apart from the scatterers, and vertically centred with them. For both cases the response was evaluated at a square grid of 25 points equally spaced $0.25 \mathrm{~m}$, and positioned $0.5 \mathrm{~m}$ away from the array of scatterers. The cylinders, with a fixed external radius of $0.1 \mathrm{~m}$, had the centres equally spaced at $0.4 \mathrm{~m}$ between them, and three thickness values were considered: $0.005 \mathrm{~m}, 0.025 \mathrm{~m}$ and $0.095 \mathrm{~m}$. Three different materials were also considered for the cylinders, all of them assumed to exhibit an isotropic elastic behaviour: PolyVinyl Chloride (PVC), concrete and steel. For all the cases, both damping and sound absorption were set to zero. The relevant material properties of the scatterers namely density, compression and shear wave speeds, are presented in Table 1. Two different fluids were also considered, namely air and water. The relevant material properties of the fluids (density and sound propagation velocity) are presented in Table 2. 


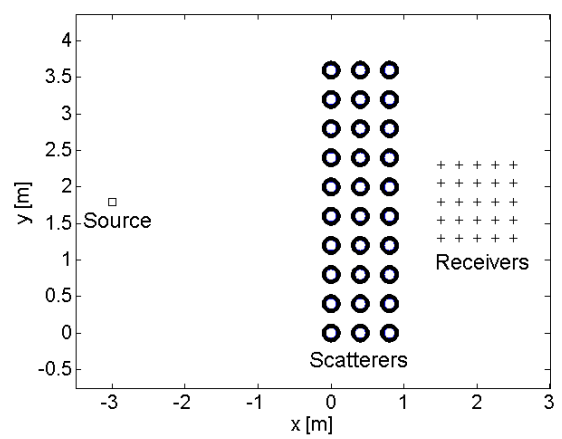

Figure 4: Scheme of the tested layout.

Table 1: Properties of the elastic materials.

\begin{tabular}{|c|c|c|c|}
\hline & $\begin{array}{c}\rho s \\
\left(\mathrm{~kg} / \mathrm{m}^{3}\right)\end{array}$ & $\alpha \mathrm{s}(\mathrm{m} / \mathrm{s})$ & $\beta \mathrm{s}(\mathrm{m} / \mathrm{s})$ \\
\hline PVC & 1400 & 2143 & 875 \\
\hline Concrete & 2400 & 3727 & 2282 \\
\hline Steel & 7900 & 5982 & 3197 \\
\hline
\end{tabular}

Table 2: Properties of the fluids.

\begin{tabular}{|c|c|c|}
\hline & $\begin{array}{c}\rho f \\
\left(\mathrm{~kg} / \mathrm{m}^{3}\right)\end{array}$ & $\alpha \mathrm{f}(\mathrm{m} / \mathrm{s})$ \\
\hline Air & 1.22 & 340 \\
\hline Water & 1000 & 1500 \\
\hline
\end{tabular}

The results for the acoustic attenuation are presented in Figure 5 when the fluid is air, and in Figure 6 for water. As can be seen, for a fluid with the properties of air, the Insertion Loss behaviour is almost independent of the cylinders stiffness, and the results provided by the two models match almost perfectly. It can be concluded that in this case the strong contrast of properties between the fluid and the solid leads to a quasi-rigid behaviour of all the tested scatterers, and the assumption of rigid boundaries would be a sufficiently good approximation.

On the other hand, in the case of water, the results provided by the two methods are quite different, and become even more pronounced if the cylinders' stiffness is reduced.

Analyzing the case of PVC scatterers, it can be seen that the Insertion Loss differs dramatically from the original model by Martins et al. [6]. Indeed, for the lower thickness of the cylinders, the computed curve reveals very small values of acoustic attenuation, and only very small and localized peaks coinciding with the resonances of the scatterers are visible. This behaviour can be seen in the pressure level wave fields presented for the $0.025 \mathrm{~m}$ thickness for a frequency away from 
the resonance peak $(500 \mathrm{~Hz}$, Figure 7a) and near it (3020 Hz, Figure 7b). As the stiffness increases, the Insertion Loss computed by the proposed model tends to increase, and for the larger thickness significant attenuations occur around $2500 \mathrm{~Hz}$. Similar conclusions can be drawn when the material is assumed to be concrete or steel (Figures $6 \mathrm{~b}$ and $6 \mathrm{c}$ ), with the attenuation increasing with the thickness of the scatterers. When steel is considered, and for the larger thickness, the Insertion Loss approaches that of the rigid model, evidencing the quasi-rigid behaviour of the scatterers in that situation.

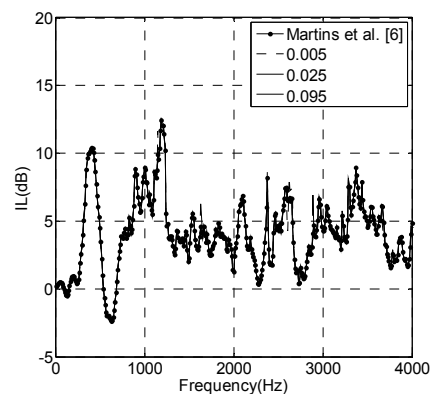

a)

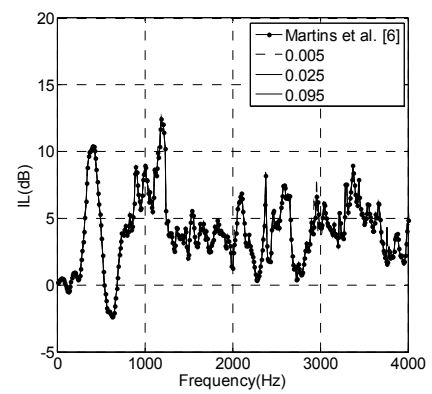

b)

Figure 5: Results of the Insertion Loss when the fluid is air, considering the cylinders to be made of: a) PVC; b) concrete.

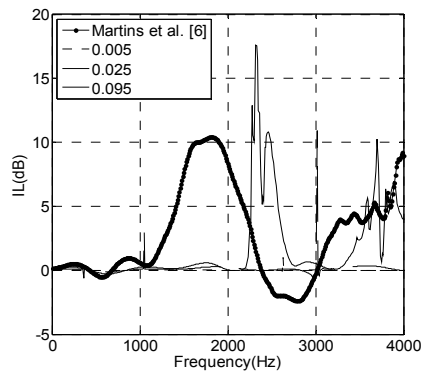

a)

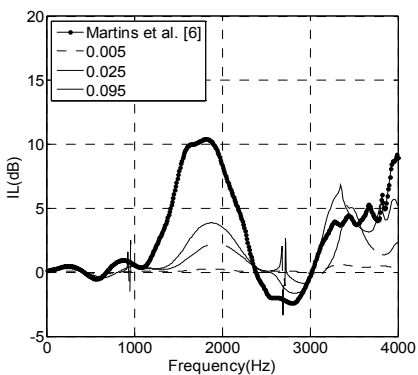

b)

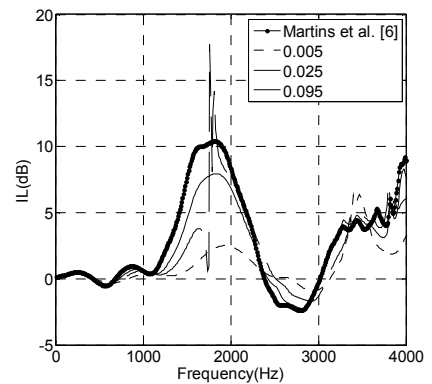

c)

Figure 6: Results of the Insertion Loss when the fluid is water, considering the cylinders to be made of: a) PVC; b) concrete; c) steel. 


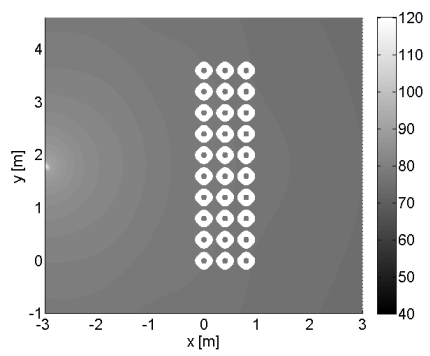

a)

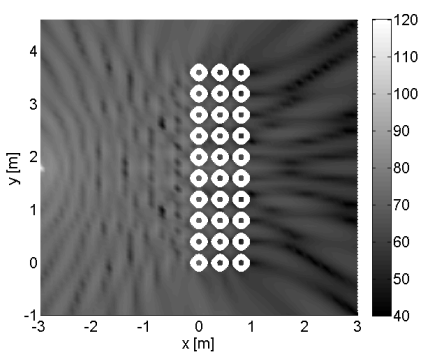

b)

Figure 7: Results of the pressure wave field for the PVC case, $0.025 \mathrm{~m}$ thick, embedded in water: a) $500 \mathrm{~Hz}$; b) $3020 \mathrm{~Hz}$.

\section{Conclusions}

In this paper, a model based on MFS, to simulate the sound propagation around a sonic crystal structure is presented. With this procedure, it is possible to avoid the discretization of the solid material and of the solid-fluid interfaces. Verification against commercial finite element model in terms of sound attenuation of these type of structures revealed excellent agreement with large computational savings.

A number of tests were performed, simulating an array of cylinders embedded within a fluid, which could be either air or water. The computed results are indicative that the effect of the elastic material of the scatterers cannot be neglected whenever the contrast between the properties of both media is not large. In the case of weak contrast between materials, such as when thin cylinders are located within water, the behaviour of the periodic structure is markedly changed, and the numerical predictions are quite different from those given by a simpler model considering rigid scatterers. If, however, a very strong contrast occurs (as when the fluid is air), the elastic cylinders behave as rigid structures, and almost no differences could be identified.

The presented results evidence the importance of correctly modelling the behaviour of the solid material when a water-embedded structure is considered, and that the fluid-structure interaction effects cannot be neglected in that case.

\section{Acknowledgements}

The research work presented herein was supported by FEDER funds through the Operational Programme for Competitiveness Factors - COMPETE and by national funds through the FCT (Portuguese Foundation for Science and Technology), under research project PTDC/ECM-COM/1438/2012. 


\section{References}

[1] Martínez-Sala R., Sancho J., Sánchez J.V., Gómez V., Llinares J. \& Meseguer F., Sound attenuation by sculpture, Nature, 378, 241, 1995.

[2] Wu L.-Y., Chen L.-W., Liu C.-M., Acoustic pressure in cavity of variously sized two-dimensional sonic crystal with various filling fraction, Phys. Lett. $A$, 373, pp. 1189-1195, 2009.

[3] Vasseur J.O., Deymier P.A, Djafari-Rouhani B., Pennec Y., HladkyHennion A-C., Absolute forbidden bands and waveguiding in twodimensional phononic crystal plates, Phys. Rev. B, 77, 085415, 2008.

[4] Sánchez-Pérez J.V., Rubio C., Martínez-Sala R., Sánchez-Grandia R., Gómez V., Acoustic barriers based on periodic arrays of scatterers, Appl. Phys. Lett., 81, 5240, 2002.

[5] Castiñeira-Ibáñez S., Rubio C., Romero-García V., Sánchez-Pérez J.V., García-Raffi L.M., Design, Manufacture and Characterization of an Acoustic Barrier Made of Multi-Phenomena Cylindrical Scatterers Arranged in a Fractal-Based Geometry, Archives of Acoustics, 37 (4), pp. 455-462, 2012.

[6] Martins, M., Godinho, L., Picado-Santos, L., Numerical Evaluation of Sound Attenuation Provided by Periodic Structures. Archives of Acoustics, 38 (4), pp. 503-516, 2013.

[7] Martins, M., Carbajo, J., Godinho, L., Mendes, P., Ramis, J., Insertion Loss Provided by a Periodic Structure - Numerical and Experimental Evaluation. Proceedings of TecniAcustica Valladolid, 2013.

[8] Godinho, L., Tadeu, A., Branco, F., Dynamic analysis of submerged fluidfilled pipelines subjected to a point pressure load. Journal of Sound and Vibration, 271(1-2), pp. 257-277, 2004.

[9] Godinho, L., Tadeu, A., Branco, F., Wave scattering by infinite cylindrical shell structures submerged in a fluid medium. Wave Motion, 38(2), pp. 131-149, 2003.

[10] Godinho, L., Tadeu, A., Mendes, P., Wave propagation around thin structures using the MFS, Computers. Materials and Continua, 5(2), pp. 117-127, 2007. 